\title{
Frank's sign with acute aortic dissection
}

\author{
Hironobu Nishiori $^{1}$, Yuichi Hirano ${ }^{1}$, Masayoshi Otsu ${ }^{1}$, and Hiroyuki Watanabe ${ }^{1}$ \\ ${ }^{1}$ Japanese Red Cross Narita Hospital
}

December 2, 2021

\begin{abstract}
A 59-year-old man with a long smoking history presented with sudden back pain. Frank's sign was noticed in his bilateral ears, and computed tomography revealed Stanford type A acute aortic dissection. If young patients have Frank's sign, attention should be paid to atherosclerotic disease including aortic disease.
\end{abstract}

\section{Frank's Sign with Acute Aortic Dissection}

${ }^{1}$ Hironobu Nishiori MD, ${ }^{1}$ Hiroyuki Watanabe MD, ${ }^{1}$ Yuichi Hirano MD,${ }^{1}$ Masayoshi Otsu MD PhD,

Division of Cardiovascular Surgery, Narita Red Cross Hospital, Chiba, Japan

Corresponding author : Hironobu Nishiori

Division of Cardiovascular Surgery, Narita Red-cross Hospital

90-1, Ida-Cho, Narita City, 286-8523, Chiba, Japan

E-mail: hironobubunishiori@gmail.com

Tel: +81-1219-7242; Fax: +81-476-22-2111

\section{Patient consent;}

Written informed consent was obtained from the patient to publish this report in accordance with the journal's patient consent policy

\begin{abstract}
A 59-year-old man with a long smoking history presented with sudden back pain. Frank's sign was noticed in his bilateral ears, and computed tomography revealed Stanford type A acute aortic dissection. If young patients have Frank's sign, attention should be paid to aortic disease in addition to coronary artery disease.

A 59-year-old man with a 40 pack-year smoking history presented our hospital with sudden back pain. He had no remarkable past medical history. The blood pressure in the left arm was $30 \mathrm{mmHg}$ higher than the right arm, and bilateral earlobes had a diagonal crease running across the earlobes at a $45^{\circ}$ angle (Frank's sign; Figure 1). A contrasted computed tomography showed Stanford type A aortic dissection (Figure 2), whereas there was no significant calcification in the coronary arteries. The patient underwent total arch replacement for aortic dissection. Although he suffered from perioperative stroke with left arm paralysis, the symptom recovered, and he was transferred to another hospital for long-term rehabilitation.

Frank's sign is caused by arteriosclerosis and is known to be associated with cardiovascular events, especially coronary artery disease, and should be paid more attention to when seen in people under the age of 60 . [1] Among the dead cases with Frank's sign, 12.3\% were reported due to aortic disease. [3] The factors contributing to atherosclerosis, such as hypertension and smoking, are also strong risk factors for aortic
\end{abstract}


disease, including aortic dissection. Frank's sign in young people, in addition to coronary artery disease, the aortic disease should be noted.

\section{CONFLICT OF INTEREST}

The authors have no pertinent conflicts of interest to report for this manuscript

\section{AUTHOR CONTRIBUTION}

HN, YH, MO and HW: cared for the patient and participated in the surgery. HN: got the patient consent form and prepared the clinical picture and computed tomography imaging data, and wrote the report. HW: read and approved the final version of the report.

\section{INFORMED CONSENT}

We have obtained the consent of the patient for publication.

\section{REFERENCE}

1. Griffing G. Frank's sign. N Engl J Med. N Engl J Med. 2014;370(10):e15.

2. Kirkham N, Murrells T, Melcher DH, Morrison EA. Diagonal earlobe creases and fatal cardiovascular disease: a necropsy study. Br Heart J. 1989;61(4):361-364. 


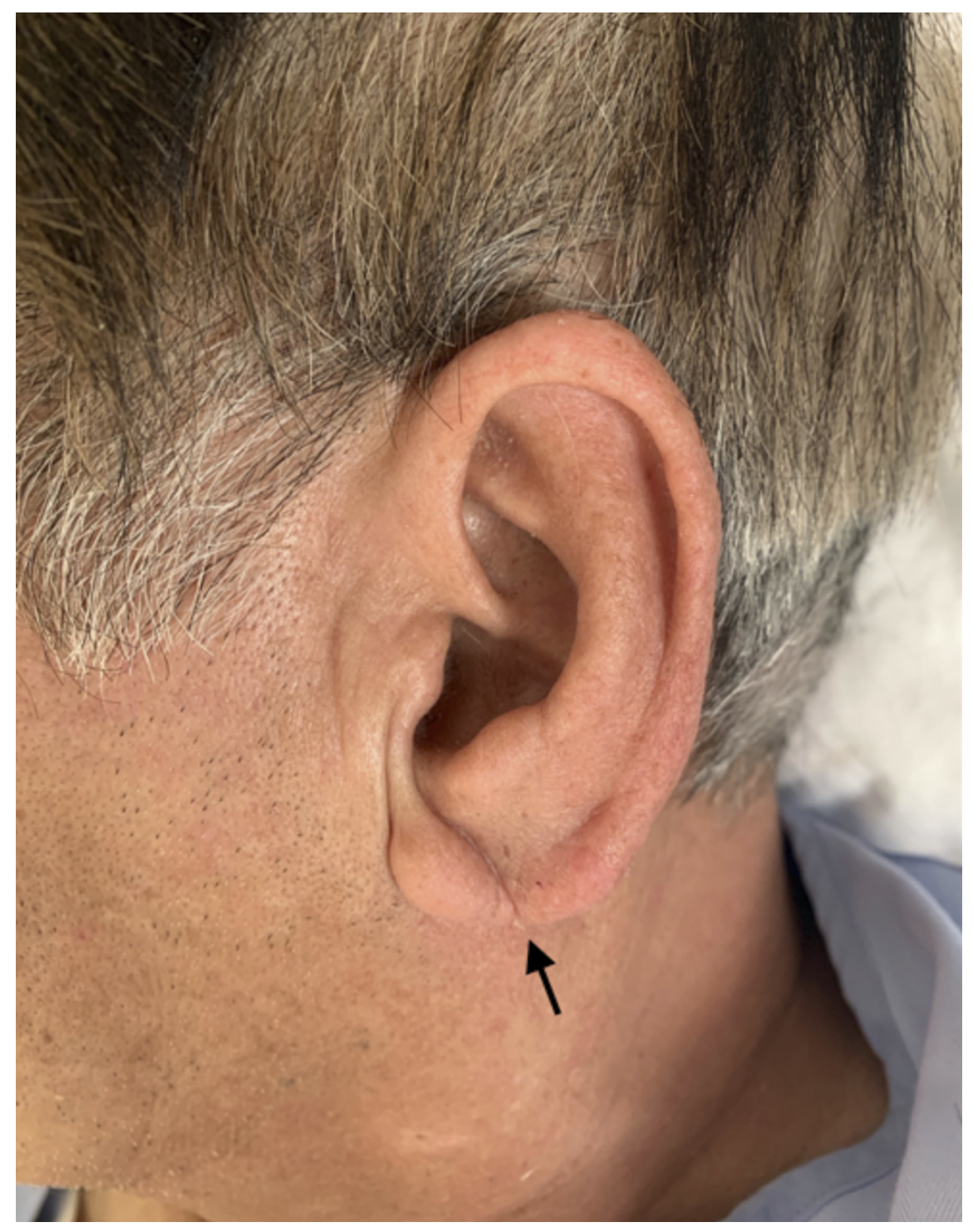




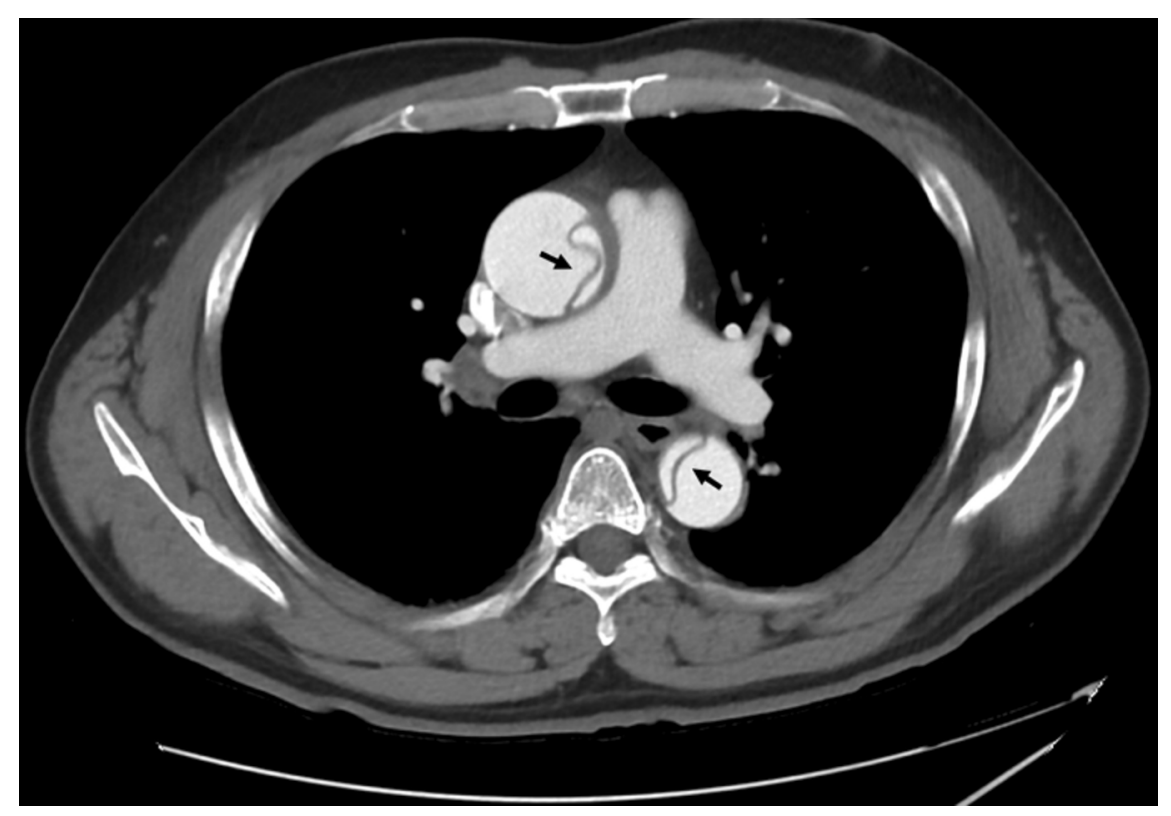

